\title{
Towards the Structural Optimization of Bladed Components Featuring Contact Interfaces
}

\author{
Julien Lainé ${ }^{1}$, Elsa Piollet ${ }^{1}$, Alain Batailly ${ }^{1}$
}

\begin{abstract}
In order to maximize their efficiency, modern aircraft engines feature reduced nominal clearances between rotating (such as bladed disks) and static (casing) components. As a consequence, structural contacts between these components are now more likely to occur and must be accounted for as early as in the design stage of the engine. To this day, there exists no relevant criterion to discriminate contacting components, such as blades, according to their sensitivity to contact events. In a recent study, it was found that a redesigned blade, featuring significant improvements with respect to its vibratory response following structural contacts, essentially differed from the original design with respect to its clearance consumption - a quantity that characterizes the evolution of blade/casing clearance as the blade vibrates over its first free-vibration modes. From this observation, it was decided to carry out a thorough investigation on the possible relation between clearance consumption and the nonlinear vibratory response following structural contacts. This paper presents an automated structural optimization procedure of a blade design using an objective-function related to the blade's clearance consumption. Thus, optimized blades feature a lower clearance consumption. By means of an in-house numerical tool dedicated to the simulation of structural contact events with a surrounding casing, it is found that optimized blades feature lower amplitudes of vibration following contacts in comparison with the original blades. Overall, it is evidenced that the blade sensitivity to contact has been lowered as certain critical speeds vanish for the optimized profile. The current paper aims at establishing a proof-of-concept and thus only considers structural aspects. Aerodynamic considerations that are key for designing efficient blades are purposely left aside in order to focus on the feasibility of blade dynamics optimization.
\end{abstract}

Keywords

rotor/sator interaction; blackbox optimization; blade design; nonlinear dynamics; clearance consumption

1 - Department of Mechanical Engineering, École Polytechnique de Montréal, P.O. Box 6079, Succ. Centre-Ville, Montréal, Québec, Canada H3C 3A7 


\title{
Vers l'optimisation structurelle de composants aubagés avec interfaces de contact
}

\author{
Julien Lainé ${ }^{1}$, Elsa Piollet ${ }^{1}$, Alain Batailly ${ }^{1}$
}

\begin{abstract}
Résumé
Afin de maximiser leur efficacité, les moteurs d'avion récents présentent des jeux réduits entre les parties tournantes (comme les disques aubagés) et fixes (carter). Ceci augmente la fréquence des contacts aubes/carter, qui doivent être pris en compte dès la conception du moteur. À ce jour, il n'existe pas de critère pertinent qui permette de prédire si une aube sera sensible aux évements de contact. Une étude récente a montré qu'une aube présentant une sensibilité significativement réduite après reconception était particulièrement différente de l'aube initiale en termes de sa consommation de jeu - une quantité qui caractérise l'évolution du jeu aube/carter lorsque l'aube vibre selon l'un de ses premier modes propres. À partir de cette observation, une recherche approfondie a été menée sur la possible relation entre la consommation de jeu et la réponse vibratoire suite à des contacts structurels. Ce papier présente une procédure d'optimisation structurelle automatisée pour la conception d'aubes en utilisant une fonction-objectif liée à la consommation de jeu de l'aube. Ainsi, les aubes optimisées présentent une consommation de jeu réduite. Un outil numérique interne dédié à la simulation de contacts aubes/carter permet d'observer que les aubes optimisées montrent des amplitudes de vibration réduites après contact avec le carter, par rapport aux aubes initiales. De manière générale, la sensibilité des aubes au contact est réduite: en effet, certaines vitesses critiques disparaissent pour l'aube optimisée. Le but de ce papier est de servir de preuve de concept, et seuls les aspects structurels sont donc considérés. Les aspects aérodynamiques, qui sont essentiels à la conception d'aubes efficaces, sont volontairement exclus afin de se concentrer sur la faisabilité de l'optimisation de la dynamique d'aube.
\end{abstract}

Mots-clés

interaction rotor/stator; optimisation boîte noire; conception d'aube; dynamique non-linéaire; consommation de jeu

1 - Département de génie mécanique, École Polytechnique de Montréal, P.O. Box 6079, Succ. Centre-Ville, Montréal, Québec, Canada H3C 3A7 


\section{Nomenclature}

\section{Acronyms}

1B first bending mode

$1 \mathrm{~T}$ first torsional mode

2B second bending mode

LE leading edge

TE trailing edge

\section{Symbols}

$s \quad$ similarity score

$c_{c} \quad$ clearance consumption

$\mathbf{u}, \phi \quad$ displacement fields

$f \quad$ objective-function

$\Gamma \quad$ optimization constraints

$\theta \quad$ iteration index

$\delta \quad$ amplitude coefficient of the blade vibration

$\Omega \quad$ angular speed

$\zeta \quad$ displacement criterion

$A \quad$ profile area

K $\quad$ stiffness matrix

f force vector

$R \quad$ blade radius

$\mathrm{x}, \mathrm{y}, \mathrm{z}$ cartesian coordinates, blade coordinate system

x', y', z' cartesian coordinates, profile coordinate system

$\bar{d} \quad$ mean distance between data points and model

$\delta p \quad$ perimeter difference

\section{Subscripts}

$p \quad$ profile number

b blade

Mathematics

$\mathcal{C}^{1}$-continuity continuity and continuity of the first derivative 


\section{Introduction}

Reducing aircraft engine fuel consumption is a major concern for manufacturers, both from economic and environmental standpoints. There are currently two avenues on which designers and researchers focus their efforts: (1) the development of new, lighter materials and (2) the reduction of operating clearances between rotating and static components. While the latter yields very significant performance improvements, it increases the probability of structural contacts between the engine components. These contacts generate blade and/or carter vibrations which can decrease the lifespan of the components through increased stress levels and high cycle fatigue. The generated vibrations are strongly nonlinear, and cannot be predicted by the linear theories still used today for the design of bladed components. The increased likelihood of potentially damaging interaction phenomena such as rubbing [1, 2], modal interaction [3] or whirling motions [4] calls for the integration of nonlinear structural considerations as early as in the design stage of bladed components. In particular, rubbing phenomena that typically involve a single blade, and which are characterized by repeated contacts between a blade-tip and the surrounding casing or the abradable coating deposited on it, must now be considered as part of the engine normal operating conditions.

Bladed component optimization is a field of research that has long been driven by aerodynamic considerations, be it related to fan [5], compressor [6, 7, 8, 9, 10, 11] or turbine [12, 13] stages. For most of these works, structural aspects are treated as optimization constraints (maximum mass, acceptable frequency range for the first bending mode...). For example, recent investigations [14] brought into focus the need for minimizing blade-tip/casing clearances as the blade vibrates in order to maximize aerodynamic performances. Such recommendation may appear both (1) acceptable from an aerodynamic standpoint and, (2) counter-intuitive from a structural point of view since manufacturers are sometimes forced to increase operating clearances in order to avoid potentially hazardous contact interactions. Because modern engine designs feature reduced clearances, structural contacts are now expected during engine operation. Aforementioned hazardous interactions thus become a major concern for engine manufacturers and a new balance must be found between aerodynamic and nonlinear structural considerations for bladed component design.

Recently, some researchers and manufacturers have focused on the redesign of existing bladed components accounting for nonlinear aspects such as large displacements, high cycle fatigue and damage mechanisms [15]. But, to the best of the authors' knowledge, only one publication [16] focuses on a redesign operation following structural contact events. The aftermath of this redesign operation was later on investigated in [17]. It was evidenced that the redesigned blade profile essentially differs from the original blade design with respect to its ability to maintain an almost constant blade/casing clearance as it vibrates along its first bending mode. This observation led to the definition of the clearance consumption as a possible relevant criterion in order to discriminate blades depending on their sensitivity to structural contacts.

The current investigation focuses on a structural, automated, blade optimization procedure. It uses the blade's clearance consumption as an optimization criterion. As a first approximation, aerodynamic considerations are left aside. Developing an efficient optimization procedure to reduce contact interactions is an important first step that must be uncoupled from other aspects of a blade design. In the long term, multiple objective optimization will be necessary to design actual blades with both aerodynamic and nonlinear vibration criteria. This paper presents the procedure and an application case acting as a proof-of-concept.

The first section of the paper is centered on the blade modeling procedure. An original blade profile parameterization is proposed and validated for existing blade profiles taken from the literature. In the second section, details are given about the notion of clearance consumption. The automated optimization procedure is then presented in the third section. This procedure is thoroughly validated and the emphasis is made on the use of open-source software. Finally, a selected profile is optimized with respect to the clearance consumption of the associated blade. Optimized and initial profiles are then run through an in-house tool dedicated to the simulation of contact events. Detailed results provided in the last section of the paper highlight that the optimized profile is less sensitive to contact events with the attenuation or even the elimination of some identified critical speeds. 


\section{Modeling}

An explicit parametric model of a blade is created for the purpose of this study. As shown in Fig. 1, it is composed of three two-dimensional profiles, representing the cross-section of the blade across its span (root I, mid-span II and tip III). The profiles position and orientation are given by the stacking law (-).

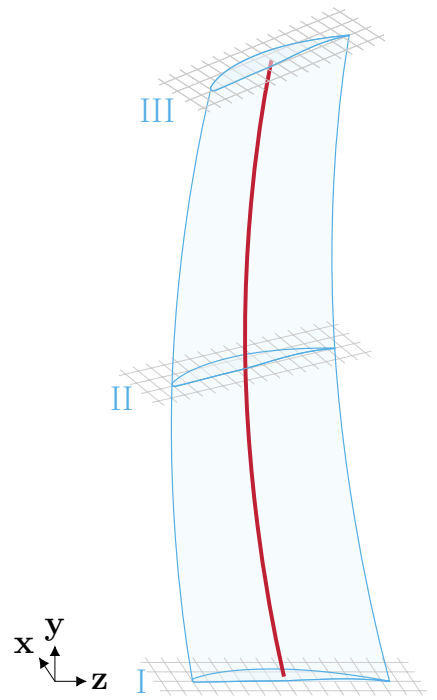

Figure 1. blade model and associated profiles

\subsection{Blade profile}

\subsubsection{Parameterization}

The proposed modeling of blade profiles, depicted in Fig. 2, builds on the work of Pritchard [18] and Cho [19]. It relies on the use of two elliptical arcs for the leading edge, one circular arc for the trailing edge, and two spline curves for the representation of both the suction side and the pressure side. This model advantageously combines a smooth representation of pressure and suction sides with an independent formulation of each side.

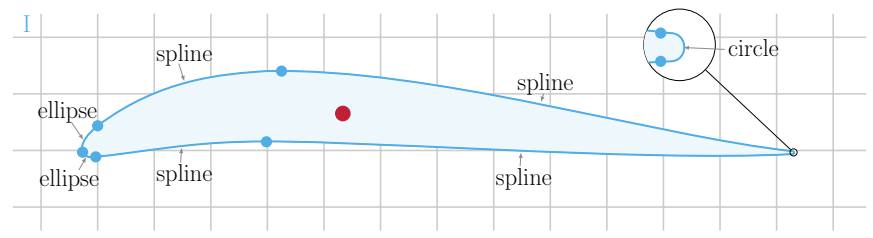

Figure 2. profile description

Such model can easily be implemented within a CAD software and it will be shown that it is flexible enough to accurately represent a large variety of existing blade profiles. As is, the model contains a total of 16 parameters. However, in order to reduce the number of parameters, the ellipses axis ratio are arbitrarily fixed to 2 for this study. In the end, the model can be entirely parameterized with only 14 parameters while ensuring $\mathcal{C}^{1}$-continuity, see Fig. 3. Note that in this paper, blade profiles are always oriented in their local coordinate system with a zero incidence, such that parameter (6) is null, and parameter (3) is the actual chord of the profile. Incidence is then applied to the profile in the global coordinate system of the blade through the stacking law discussed further in this paper.

\subsubsection{Application to existing blade profiles}

The versatility of the proposed model is assessed by applying it to well-known existing blade profiles that may be found in the literature as a list of data points or coordinates [20,21, 22]. To this end, an optimization procedure has 

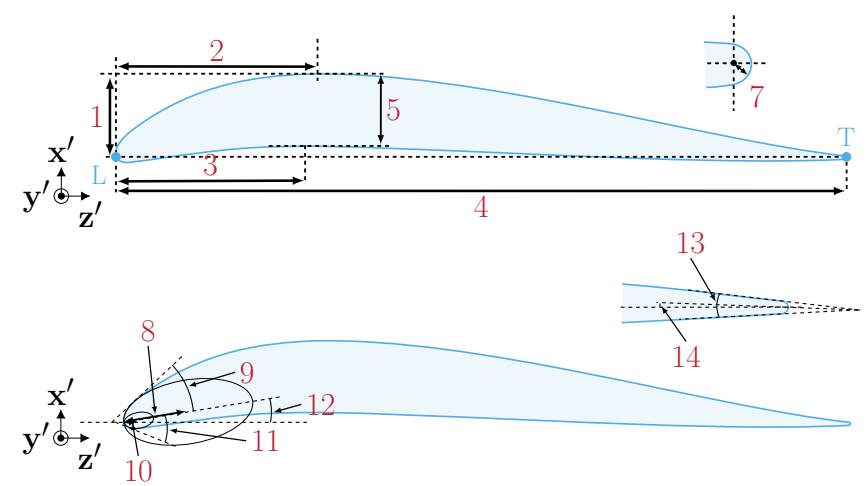
(1) suction peak point ( $x$ coordinate)
(8) major radius upper ellipse
(2) suction peak point ( $z$ coordinate)
(9) inlet upper wedge angle
(3) pression peak point ( $z$ coordinate)
(10) major radius lower ellipse
(4) axial chord $=z_{\mathrm{T}}-z_{\mathrm{L}}$
(11) inlet lower wedge angle
(5) maximum thickness
(12) blade inlet angle
(6) tangential chord $=x_{\mathrm{T}}-x_{\mathrm{I}}$
(13) outlet wedge angle
(7) trailing edge radius
(14) blade outlet angle

Figure 3. profile parameters, L: leading edge at origin and T: trailing edge

been designed in order to identify the optimal value of each of the 14 parameters for each profile of interest. This procedure is twofold:

first step: all parameters that may be obtained directly from the profile coordinates given in the literature are computed:

- (1) and (2): suction peak coordinates,

- (4): axial chord,

- (6): tangential chord.

second step: remaining parameters are identified by an optimization algorithm ${ }^{1}$ in order to fit the blade profile with the given coordinates. This fitting is estimated by a similarity score $s$ calculated with: (1) the mean distance $\bar{d}$ between the data points and the computed profile and, $(2)$ the perimeters difference $\delta p$ :

$$
s=1-(0.9 \bar{d}+0.1 \delta p)
$$

The closer $s$ is to 1 , the better the approximation of the profile. Figure 4 pictures the application of this criterion on an elliptical shape that is approximated using the proposed set of parameters. Four consecutive iterations of the optimization procedure are depicted, they feature a similarity score ranging from $s=-0.22$ to $s=0.944$. The last iteration with $s=0.944$, see Fig. $4 \mathrm{~d}$, provides a satisfying match with the targetted shape. For each iteration, the relative area error is also displayed, this value indicates the relative error between the area of the targetted ellipse and the area of the computed profile. Contrary to the relative area error, the similarity score $s$ accounts for local mismatch between the optimized profile and given data points thus making it more relevant for estimating the quality of the fitting. As an example, Figs. 4b, 4c and $4 \mathrm{~d}$ feature almost identical relative area error while the similarity score decreases significantly as the accuracy of the fitting increases.

The fitting of 6 compressor blade profiles (two NACA profiles as defined in [20], 3 profiles from the rotor 37 blade given in [21] and the man ghh 1-s1 profile from [22]) with the proposed model is depicted in Fig. 5. For each profile, there is an excellent visual match between the parameterized model and the data points given in the literature. This observation is confirmed by the very good similarity score obtained for each profile. These results underline that the

\footnotetext{
${ }^{1}$ The employed optimization strategy relies on the NOMAD software presented in details in the following of the paper.
} 


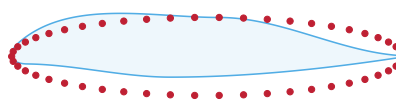

(a) $s=-0.22-\frac{d A}{A}=30 \%$

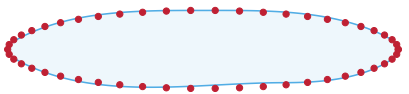

(c) $s=0.87-\frac{d A}{A}=0.5 \%$

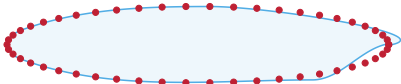

(b) $s=0.80-\frac{d A}{A}=0.8 \%$

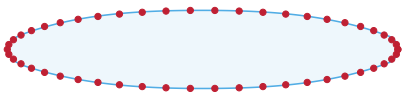

(d) $s=0.944-\frac{d A}{A}=0.6 \%$

Figure 4. similarity score and relative area errors computed for an elliptical shape $(\bullet)$ and four iterated sets of parameters

proposed model can accurately represent a large variety of blade profiles thus emphasizing the versatility of the proposed parameterization.

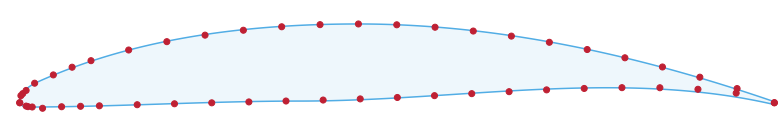

(a) NACA65-(10)10 profile [20] $-s=0.9688$

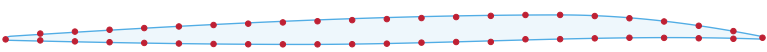

(c) rotor 37 tip profile [21] $-s=0.965$

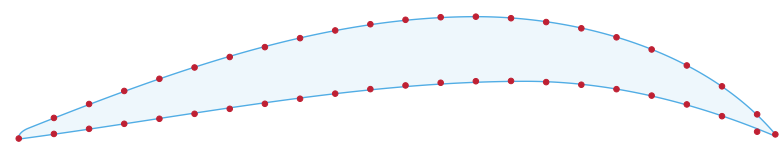

(e) rotor 37 root profile [21] $-s=0.9634$

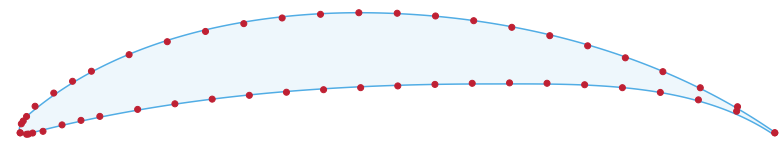

(b) NACA65-(20)10 profile [20] - $s=0.956$

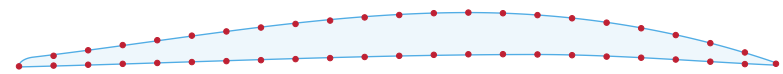

(d) rotor $37 \mathrm{mid}$ profile [21] $-s=0.9701$

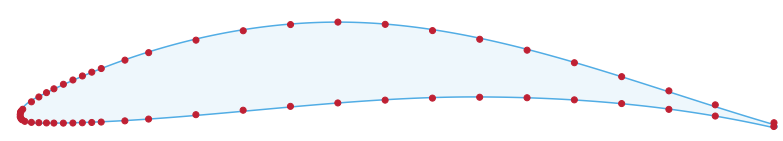

(f) man ghh 1-s1 profile [22] $-s=0.9699$

Figure 5. superimposition of 6 compressor blade profiles $(\bullet)$ as given in the literature and the associated parameterized profiles $(\square)$

\subsection{Full blade}

As mentioned above, the full blade CAD model is obtained by an extrusion from a blade profile to the next, see Fig. 1. This extrusion follows a guideline, namely the stacking law, which is composed of two elements : (1) the stacking line that connects all the profiles' centers of gravity and, (2) the stagger angle that defines the relative orientation between the profiles. The whole blade is then oriented with respect to the rotor axis by means of the angle of incidence.

\subsubsection{Stacking line}

The 3 profiles of the blade are defined in 3 parallel planes equally distributed along the blade height. Their position within those planes is given by the stacking line which is a 3D spline that intersects each of the planes at the center of gravity of the corresponding profile. Thus, the stacking line, whose parameters are defined in Fig. 6, controls the blade tilt with the lean angle (15) and the sweep angle (16) and the blade curvature with the bowed distance (17). The hub radius (18) and the blade height (19) give the radial positions of the root profile and the tip profile.

\subsubsection{Stagger angle and angle of incidence}

The stagger angle, illustrated in Fig. 7, allows for a change in the profiles' orientation, therefore twisting the blade along the radial direction $\mathbf{y}$. It is defined as the angle between the root and tip profiles. Finally, the blade is oriented 


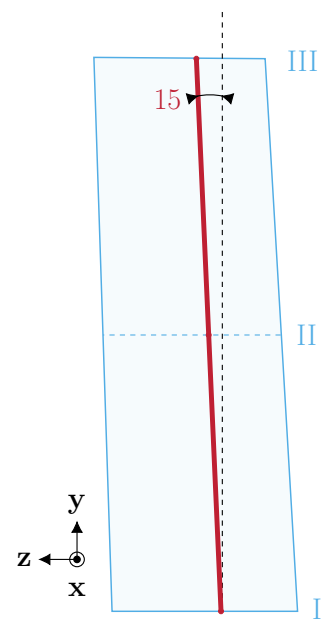

(a) side view of the blade

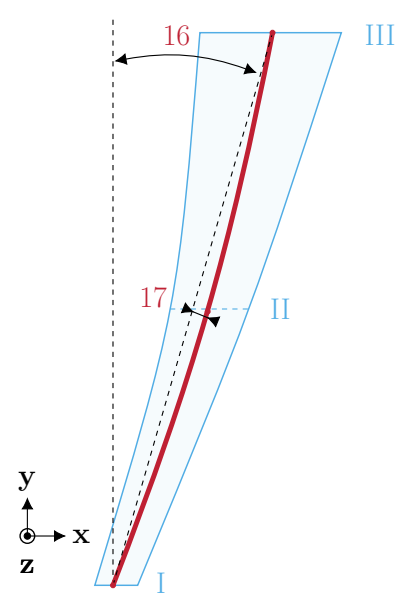

(b) rear view of the blade

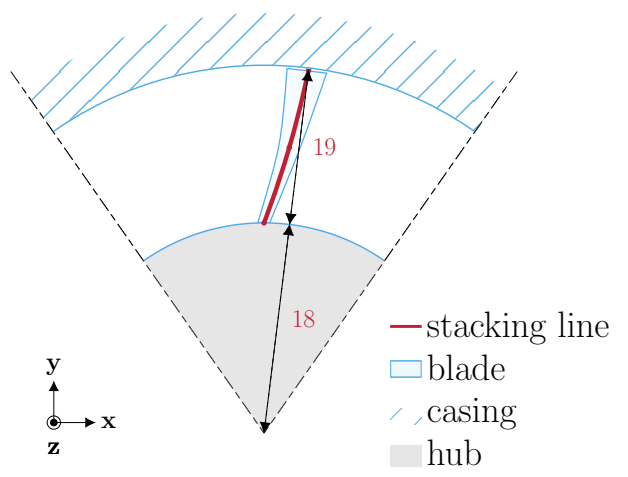

(c) rear view of the blade with the hub and the casing
(15) sweep angle
(17) bowed distance
(19) blade height
(16) lean angle
(18) hub radius

Figure 6. stacking line parametrization with 2 angles and 3 lengths

with respect to the rotor axis with the angle of incidence at the blade root. In the end $3 \times 14+7=49$ parameters are needed to build the whole blade : 14 parameters for each profile (see Fig. 3) and 7 parameters for the 3D extrusion (as shown in Figs. 6 and 7). 


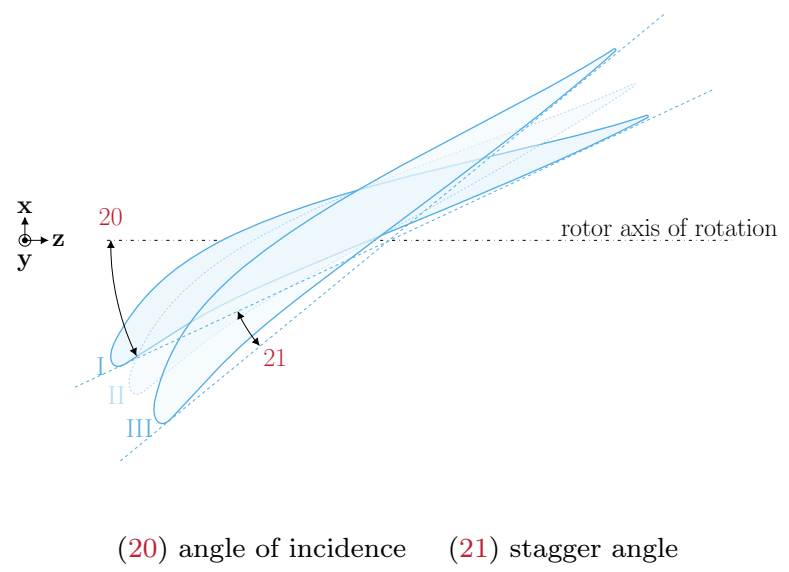

Figure 7. stagger angle and angle of incidence

\section{Clearance consumption}

\subsection{Definition}

The notion of clearance consumption $c_{c}$ was recently introduced in [17]. For a given location along the blade tip, this quantity refers to the evolution of blade/casing clearance as the blade vibrates along one of its free vibration mode, typically the first bending mode (1B) or the first torsional mode (1T). Associated clearance consumptions are respectively denoted $c_{c}^{1 \mathrm{~B}}$ and $c_{c}^{1 \mathrm{~T}}$. In this paper, as a first step towards a more general procedure, all clearance consumptions are computed at the blade leading edge and are relative to the first bending mode of the blade. Assessment of the effect of mode selection and point selection goes beyond of the scope of this paper.

As the blade vibrates, a positive clearance consumption indicates that the blade is getting closer to the casing. Conversely, a negative clearance consumption implies that the blade/casing clearance has increased. Clearance consumption is defined over an admissible domain of vibration bounded by a maximum displacement criterion $\zeta$. In this paper, the employed criterion is related to the blade's leading edge tangential displacement (along the $\mathbf{x}$ direction, see Fig. 6):

$$
\left\|u_{\mathrm{LE}, \mathrm{x}}\right\| \leq \zeta
$$

The value of $\zeta$ is discussed later in this paper.

\subsection{Computation}

A modal analysis of the blade finite element model provides the blade's first bending mode $\phi_{1 \mathrm{~B}}$. This mode is scaled in order to reach the limit of the aforementioned criterion:

$$
\phi_{1 \mathrm{~B}}= \pm \zeta
$$

A static computation then allows retrieving the force vector $\mathbf{f}_{1 \mathrm{~B}}$ which would yield the displacement field $\phi_{1 \mathrm{~B}}$ :

$$
\mathbf{f}_{1 \mathrm{~B}}=\mathbf{K}_{\mathrm{b}} \phi_{1 \mathrm{~B}}
$$

where $\mathbf{K}_{\mathrm{b}}$ is the blade's stiffness matrix. Then, for $\delta \in[-1 ; 1]$, several displacement fields $\mathbf{u}_{1 \mathrm{~B}}$ are computed:

$$
\mathbf{u}_{1 \mathrm{~B}}(\delta)=\delta \mathbf{K}_{\mathrm{b}}^{-1} \mathbf{f}_{1 \mathrm{~B}}
$$

For each $\mathbf{u}_{1 \mathrm{~B}}(\delta)$, the clearance consumption $c_{\mathrm{c}}(\delta)$ is then calculated as follows:

$$
c_{\mathrm{c}}(\delta)=R(\delta)-R_{0}
$$




$$
R(\delta)=\sqrt{(x(\delta))^{2}+(y(\delta))^{2}}
$$

where:

- $x(\delta)$ and $y(\delta)$ are the cartesian coordinates of the leading edge extracted from the blade displacement field $\mathbf{u}_{1 \mathrm{~B}}(\delta)$;

- $R_{0}$ represents the radial coordinate of the blade's leading edge at rest, see Fig.8.

Figure 9 illustrates the clearance consumption as a function of $\delta$. One may note that, depending on the casing conicity, the computation of the clearance consumption could involve the axial displacement of the blade. In the present study, all casings are assumed perfectly cylindrical for the sake of simplicity.

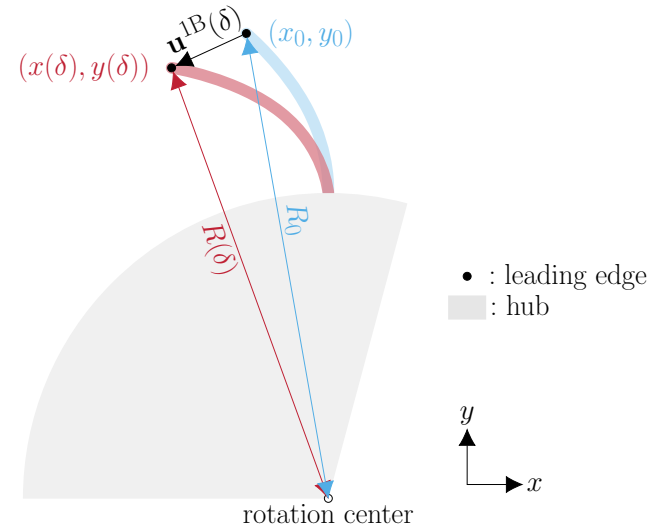

Figure 8. blade radius calculation, blade at rest $(-)$ and vibrating blade $(-)$

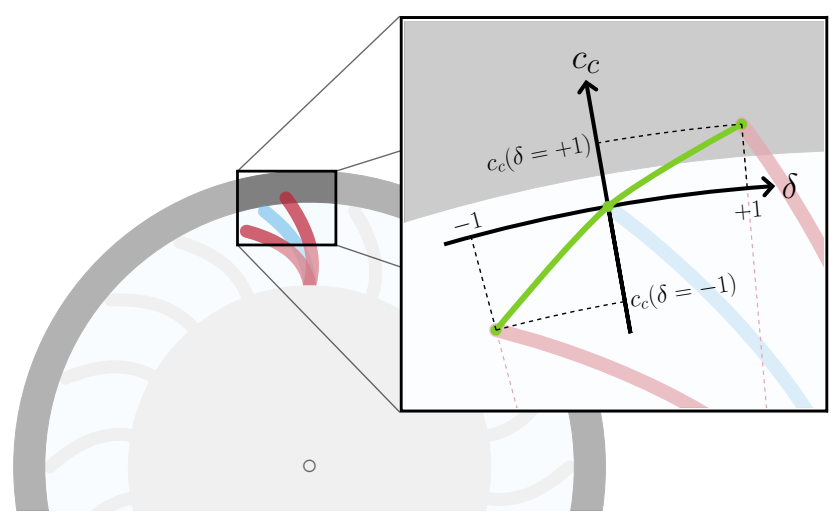

Figure 9. schematic representation of a blade's clearance consumption ( $=$ ), blade at rest $(\square)$ and vibrating blade $(\square)$

\section{Optimization procedure}

The goal of the optimization procedure is to update a blade geometry in order to minimize its leading edge clearance consumption as it vibrates along the first bending mode. Given the clearance consumption of the blade, see graph $(-)$ in Fig. 9 for instance, the procedure aims at flattening the graph with respect to the abscissa axis, i.e. it minimizes the value:

$$
f(\theta)=\max _{\delta \in\{-1,1\}}\left(c_{\mathrm{c}}(\delta)\right)
$$


at each iteration step $\theta$.

This value is the objective-function $f$ that is minimized by the optimization algorithm. The optimization also features 3 constraints $\Gamma_{p}, p=1,2,3$ to keep the area of the 3 profiles within a $\pm 10 \%$ range around the initial areas in order to preserve realistic blade profiles:

$$
\Gamma_{p}=-0.1+\frac{\left|A_{p}-A_{p, 0}\right|}{A_{p, 0}}
$$

where $A_{p}$ stands for the area of the iterated profile $p$, and $A_{p, 0}$ stands for the area of the initial profile $p$ (constraints that are negative are considered satisfied by the employed optimization algorithm).

As a first step towards an automated blade design procedure, the set of constraints $\Gamma_{p}$ is voluntarily not too restrictive. In future work, the development of this set of constraints through, for instance, the inclusion of aerodynamic considerations, will allow for the design of industrially relevant bladed components.

\subsection{Optimization algorithm}

The optimization is conducted with the software NOMAD [23, 24] and the Mesh adaptive direct search (MADS) algorithm. NOMAD is a blackbox optimization software, which means the objective-function and the constraints are given at each iteration by an external computer code: the black box. The algorithm is totally independent from the black box internal operations and only uses the objective-function and constraints values at each iteration.

\subsection{Inside the black box}

The iteration $\theta$ of the optimization procedure, during which the objective-function and the constraints are computed, is depicted in Fig.10. The set of 49 parameters obtained by NOMAD is used to build the blade CAD model with

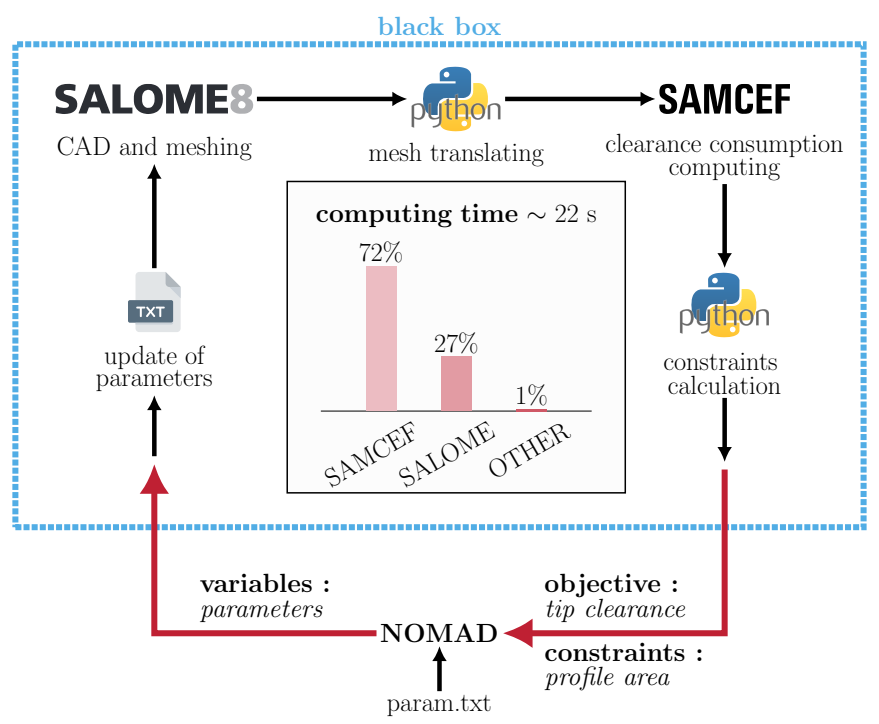

Figure 10. description of one iteration of the procedure

the SALOME software package [25], an open-source platform that provides both CAD and meshing tools. Within SALOME, the computation of each profile area allows for the estimation of the three constraints $\Gamma_{p}(\theta)$. The CAD model is then meshed with hexahedric elements with two fixed parameters: (1) the number of elements along the blade thickness and, (2) the total number of elements in the mesh. The resulting finite element mesh is read by the commercial finite element solver Samcef (LMS Samtech) in order to compute the blade's clearance consumption, specifically $c_{c}(\delta=+1)$ and $c_{c}(\delta=-1)$. From these two values, the objective-function defined in Eq. (8) may then be computed. 
The objective function $f(\theta)$ and the three constraints $\Gamma_{p}(\theta)$ are finally returned to NOMAD that will provide a new set of design parameters for iteration $\theta+1$.

\section{Validity domain and spatial convergence}

In order to ensure a meaningful optimization, it is critical to make sure that clearance consumptions are accurate. The use of a linear solver within the Samcef software allows for significantly reduced computation times. However, obtained results may differ from those obtained with a nonlinear solver, depending on the blade vibration amplitude which is controlled by the $\zeta$ parameter, see Eq. (2). Therefore, this section focuses on the definition of an acceptable value of $\zeta$. A NACA65-(10)10 profile is considered, along with the design parameters mentioned in Tab. 1. Figure 11

\begin{tabular}{|c|c|}
\hline $\begin{array}{l}\text { (15) } \text { sweep angle } \\
\text { (16) lean angle } \\
\text { (17) bowed distance } \\
\text { (18) hub radius } \\
\text { (19) blade height } \\
\text { (20) angle of incidence } \\
\text { (21) } \\
\text { stagger angle }\end{array}$ & $\begin{array}{c}-7.5^{\circ} \\
20^{\circ} \\
20 \mathrm{~mm} \\
350 \mathrm{~mm} \\
150 \mathrm{~mm} \\
0^{\circ} \\
10^{\circ}\end{array}$ \\
\hline
\end{tabular}

Table 1. additional design parameters

shows the clearance consumption — at the leading edge of the blade - computed with both linear $(-)$ and nonlinear (-- - ) solvers. Calculations are run with a 5000 element mesh of the blade. For the sake of example, a fairly large value of $\zeta$ is considered: $\zeta=25 \mathrm{~mm}$. Such displacement is equal to $17 \%$ of the blade height. Figure 11

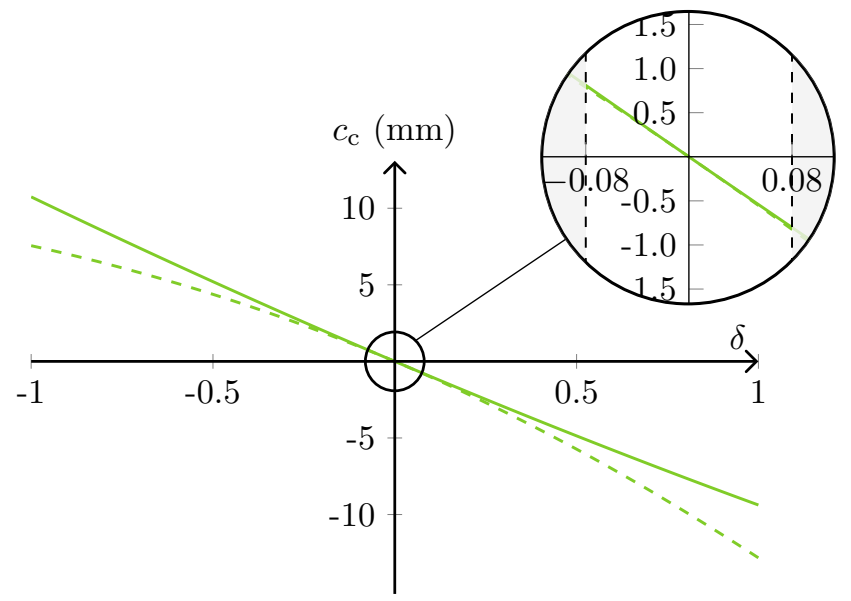

Figure 11. clearance consumption of the blade on the first bending mode for $\zeta=25$ mm with linear $(-)$ and nonlinear $(---)$ solvers, zoom over $\delta \in[-0.08 ; 0.08]$

underlines that the difference between linear and nonlinear calculations increases with $\delta$. A zoom over the domain $\delta \in[-0.08 ; 0.08]$ indicates that, over this domain, linear and nonlinear results are perfectly superimposed. As $\delta=0.08$ corresponds to a tangential displacement $\zeta=2 \mathrm{~mm}$, this value of $\zeta$ will be considered throughout this paper.

Finally, two other parameters are investigated: the total number of elements in the mesh and the number of elements over the blade thickness. It is standard practice in the industry to consider a minimum of two elements over the blade thickness. For this study, this number is set to four. Figure 12 shows the evolution of the maximum value of the clearance consumption depending on the total number of elements. The calculations converge rapidly: past 500 elements the value of clearance consumption remains almost identical from a mesh to another. In the following, 1000 elements are used for all blade meshes. 


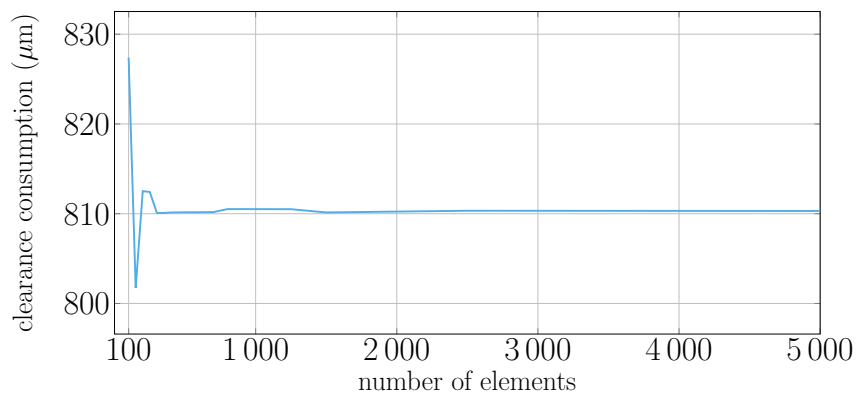

Figure 12. convergence of the clearance consumption with the number of finite elements

It may be useful to comment on the physical meaning of the amplitude levels encountered in the following simulations. As will be shown, amplitudes of vibration where $5 \mathrm{~mm}$ of abradable coating are fully worn off in the radial direction can be observed at the end of contact simulations. While such levels of vibration may seem extreme, they can indeed be encountered in industrial applications. Moreover, the numerical strategy used in this paper was shown to provide results that agree qualitatively with experimental testing on full scale rotors [2]. It should be kept in mind, however, that this numerical strategy is intended to be used to identify the start of contact interactions, and does not take into account the nonlinearities that may arise with large displacements.

\section{Application to a NACA65-(10)10 profile}

\subsection{Initial blade model analysis}

As a proof-of-concept, a generic NACA65-(10)10 profile is considered in this application. In order to reduce the number of parameters, the three profiles are assumed identical along the blade span. This yields a total of 21 parameters (14 for the blade profile and 7 for the extrusion). The profile of interest is shown in Fig. 13.

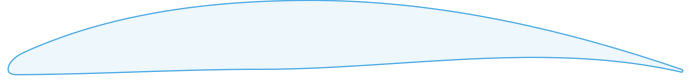

Figure 13. initial profile: NACA65-(10)10

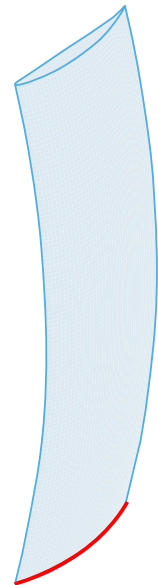

Figure $14 . \quad$ iniclamped area $(-)$ tial blade model,

The associated 3D model is depicted in Fig. 14. The mechanical properties of the blade material are summed up in Tab. 2. This blade model is meshed and with hexahedric finite elements, as described above, and the following boundary conditions are applied: the blade foot is clamped ( - ), see Fig. 14. Additionally, eight nodes are retained along the blade chord in order to manage potential contacts with the surrounding casing. Based on the numerical strategy presented in [2], and not recalled here for the sake of brevity, a reduced-order model of the blade is computed. This model is then used for carrying out nonlinear contact simulations between the blade rotating within a distorted casing. The casing distortion used in this study is an ovalization. All numerical validations in terms of time and space convergence (respectively with respect to the duration of the time integration step and spatial discretization parameters such as the modal reduction parameter) have been checked prior to running the interaction simulations.

Contact simulations involve a deformed - yet perfectly rigid — casing on which is deposited an abradable coating. Centrifugal effects are accounted for and the initial bladetip/casing clearance is set to $0.5 \mathrm{~mm}$ from the leading edge to the trailing edge. The abradable coating is $5 \mathrm{~mm}$ thick. The blade undergoes 100 revolutions at constant angular speed $\Omega$. Simulations are run for $\Omega \in[28 ; 63] \mathrm{Hz}$. Over this angular speed range, a modal analysis of the blade allows for a prediction of critical speeds, as depicted in the Campbell diagram in Fig. 15. Potential critical speeds, some of which are marked with the symbols $\mathbf{\Delta}, \mathbf{-}, \bullet$, and $\bullet$, correspond to an 


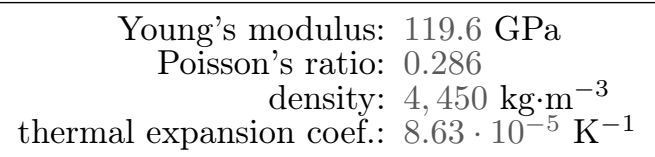

Table 2. blade material's mechanical properties

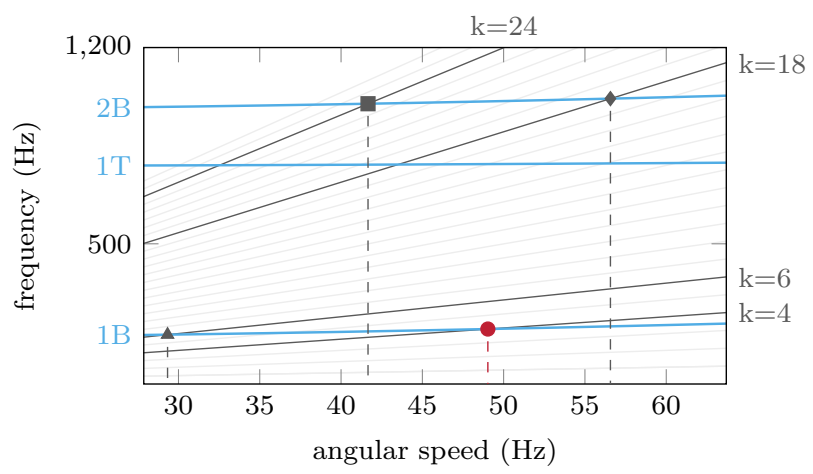

Figure 15. Campbell diagram of the inital blade

intersection between one of the blade's eigenfrequencies and a specific engine order $k$. Because of the intrinsically nonlinear nature of the simulated interaction, it is not possible to predict beforehand which of these interaction points will be critical. At the end of each contact simulation, the wear pattern predicted within the abradable coating is retreived. This pattern is plotted for each value of $\Omega$ using a colour code from white (no wear) to black (maximum wear); areas where the abradable coating is fully worn off are depicted in red. The juxtaposition of these wear profiles yields the wear map shown in Fig. 16. This wear map provides useful insight on the blade's dynamics depending on the angular speed $\Omega$. Several interactions are found, matching a specific number of lobes worn out within the abradable coating. Throughout the angular speed range of interest, one may note two privileged wear areas along the casing's circumference around $\pi / 2$ and $3 \pi / 2$ in both Figs. 16 and 20. These areas are related to the fact that the casing is distorted along an oval shape yielding two privileged contact areas as a consequence. Thus, these wear areas are expected and do not reveal anything with respect to the blade's dynamics: they are found in

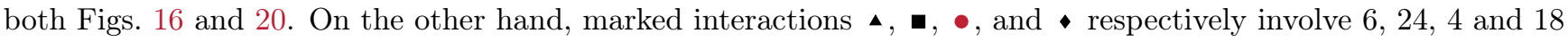
wear lobes. As shown in Fig. 15, they can be associated with a resonance along a specific free-vibration mode of the blade. However, further analysis of the blade dynamics goes beyond the scope of this study.

\subsection{Blade optimization}

As mentioned above, the blade optimization is carried out assuming that the three profiles are identical along the blade's span. In addition, three of the 14 profile parameters are fixed during the optimization procedure: (7) the trailing edge radius which is assumed to have little impact on the blade's structural behavior, and both (4) the axial and (6) tangential chords to keep the same profile length. All other profile parameters are bound within a $\pm 20 \%$ variation range. Finally, all extrusion parameters are constant but (17) the bowed distance that may vary within a $\pm 20 \%$ variation range.

Initial and optimized values of each parameters are given in Tab. 3. Converged optimized values are obtained after 1500 iterations. Initial and optimized profiles are superimposed in Fig. 17. As underlined in the introduction of the paper, this optimization is carried out without considering aerodynamics. Only the set of constraints $\Gamma_{p}$ applies thus making the optimized profile (-) acceptable. Logically, the clearance consumption of the optimized profile is lower than the one of the initial profile, see Fig. 18. The maximum value of the clearance consumption is lowered by $32 \%$ from $0.155 \mathrm{~mm}$ to $0.105 \mathrm{~mm}$. The Campbell diagram of the optimized blade is depicted in Fig. 19. A brief 


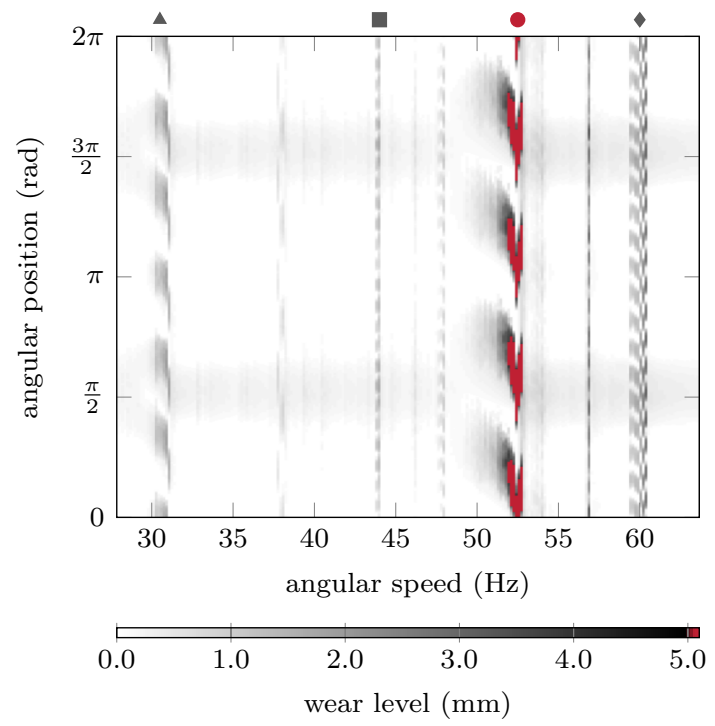

Figure 16. wear map obtained with the initial blade

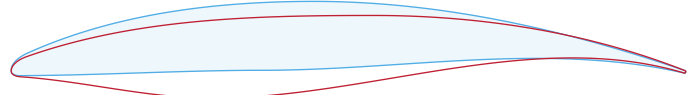

Figure 17. superimposition of initial ( - ) and optimized $(-)$ profiles

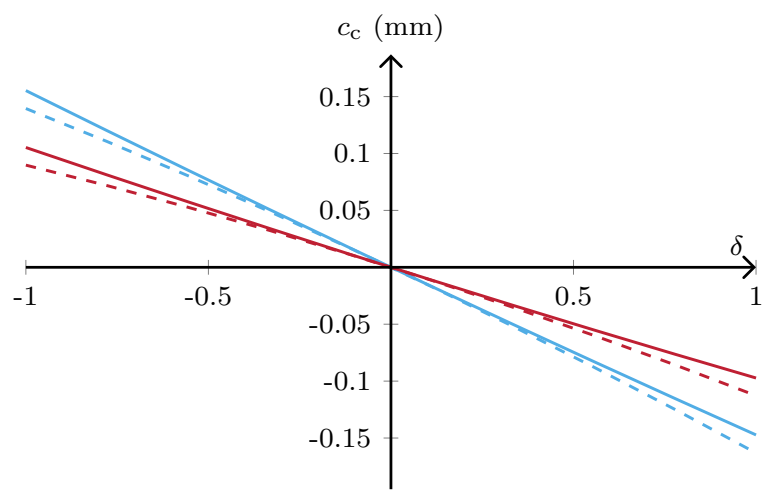

Figure 18. clearance consumption of initial (-) and optimized (-) blade models on their first bending mode, non-linear results are given by the dashed lines

comparison between Figs. 15 and 19 indicates that the optimization of the blade led to a significant increase of its second and third free-vibration frequencies respectively related to the first torsional (1T) and the second bending (2B) modes.

\subsection{Optimized blade model analysis}

Same as for the initial blade model, the optimized blade model is used for nonlinear contact simulations. Simulation parameters are identical to those previously used: the blade undergoes 100 revolutions within a distorted casing in order to initiate contact. The wear map obtained with the optimized profile is depicted in Fig. 20. Interactions marked $(\mathbf{\bullet}, \mathbf{\bullet}, \bullet, \bullet)$ in Fig. 19 are directly related to the blade intrinsic vibration behavior. 


\begin{tabular}{|c|c|c|}
\hline parameters & initial & optimized \\
\hline $\begin{array}{r}\text { suction peak point (X) }(1) \\
\text { suction peak point }(\mathrm{Z})(2) \\
\text { pression peak point }(\mathrm{Z})(3) \\
\text { axial chord }(4) \\
\text { maximum thickness }(5) \\
\text { tangential chord }(6) \\
\text { trailing edge radius }(7) \\
\text { major radius upper ellipse } \\
\text { inlet upper wedge angle }(9) \\
\text { major radius lower ellipse }(10) \\
\text { inlet lower wedge angle }(11) \\
\text { blade inlet angle }(12) \\
\text { outlet wedge angle }(13) \\
\text { blade outlet angle }(14) \\
\text { sweep angle }(15) \\
\text { lean angle }(16) \\
\text { bowed distance }(17) \\
\text { hub radius }(18) \\
\text { blade height }(19) \\
\text { angle of incidence }(20) \\
\text { stagger angle }(21)\end{array}$ & $\begin{array}{c}5.22 \mathrm{~mm} \\
22.44 \mathrm{~mm} \\
18.599 \mathrm{~mm} \\
50.0 \mathrm{~mm} \\
5.108 \mathrm{~mm} \\
0.0 \mathrm{~mm} \\
0.1 \mathrm{~mm} \\
2.544^{\mathrm{mm}} \\
13.824^{\circ} \\
0.835 \mathrm{~mm} \\
10.238^{\circ} \\
10.459^{\circ} \\
6.072^{\circ} \\
15.945^{\circ} \\
0 \mathrm{~mm} \\
0 \mathrm{~mm} \\
10 \mathrm{~mm} \\
350 \mathrm{~mm} \\
150 \mathrm{~mm} \\
0 \mathrm{~mm} \\
0 \mathrm{~mm}\end{array}$ & 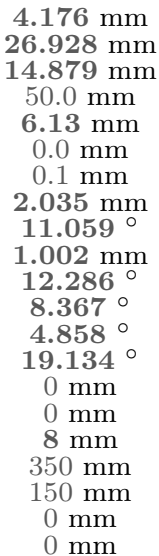 \\
\hline
\end{tabular}

Table 3. initial and optimized parameters of the investigated blade model

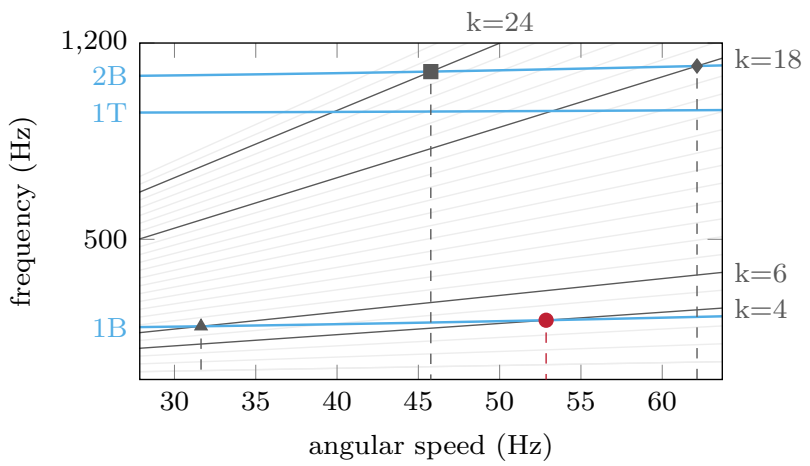

Figure 19. Campbell diagram of the optimized blade

From Fig. 16 to Fig. 20, many interactions vanish as evidenced by the absence of significant wear predicted within the abradable coating. In particular, previously identified interactions $\mathbf{a}$ and are not observed for the optimized blade, and all critical areas disappear in the angular speed range $\Omega \in[35 ; 52] \mathrm{Hz}$ which indicate a reduction in blade vibration amplitude. Only two critical speeds are found in common between the two wear maps: they are 1B related interactions $\wedge$ and $\bullet$. Numerical results thus suggest that the optimized blade's response following contact events features significantly lower amplitudes than the initial blade. This is illustrated on Fig. 21 which compares the time responses corresponding to interaction - between the two blades: time histories of each blade's leading edge radial displacement are detailed over 2 seconds. This interaction corresponds to a rotational speed of $44 \mathrm{~Hz}$ for the initial blade. For the final blade, a comparison between both Campbell diagrams (Fig. 15 and Fig. 19) and both wear maps (Fig. 16 and Fig. 20) indicate a corresponding rotational speed of $47 \mathrm{~Hz}$. Fig. 21 illustrates clearly the fact that the interaction was suppressed: the optimized blade response has a lower amplitude of under $0.2 \mathrm{~mm}$, and reaches a steady-state response around $0.8 \mathrm{~s}$. The original blade on the other hand shows a diverging response with amplitudes higher than $2.5 \mathrm{~mm}$ at the end of the 100 cycles.

On the other hand, Fig. 22 gives a closer look at the blades' dynamics at interaction point $\bullet$. It is evidenced that, in both cases, the abradable coating is fully worn off: the predicted wear level reaches the maximum value of $5 \mathrm{~mm}$ (the initial thickness of the coating) in both wear maps. In agreement with this observation, both blades feature radial displacements reaching a maximum value in the vicinity of $5 \mathrm{~mm}$ in Fig. 22.

In terms of robust design, the disappearance of all critical areas over the angular speed range $\Omega \in[35 ; 52] \mathrm{Hz}$ 


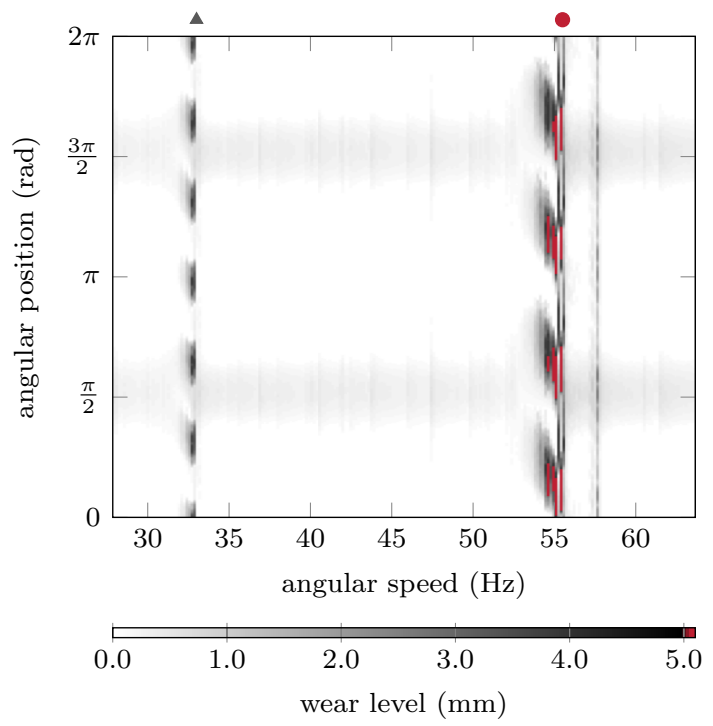

Figure 20. wear map obtained with the optimized blade

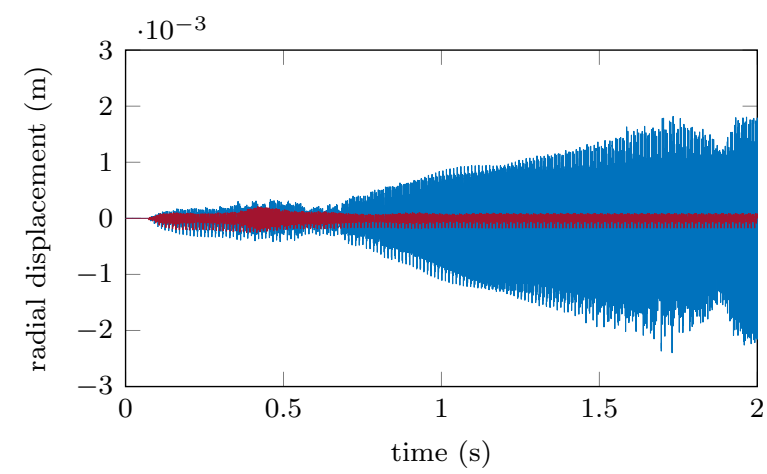

Figure 21. time history of each blade's leading edge radial displacement for interaction point $\boldsymbol{\square}$ : initial blade ( $\longrightarrow$ ) and optimized blade $(-)$

seems highly interesting. While these results must still be confirmed for a broader range of contact configurations, the improvements observed between the initial and optimized blade models provide promising elements with respect to the relevance of the use of clearance consumption in order to design bladed components robust with respect to contact interactions.

The employed numerical strategy allows for more in-depth analyses including the computation of stress fields within the blades, which will be useful in future applications of the procedure. In the particular case of the optimization carried out in this paper, Fig. 23 shows the stress field in the blade for interaction $\bullet$. The numerical strategy employed for contact simulation leads to high force levels applied on specific nodes, which yield artificially high stress levels on some nodes at the blade tip. Therefore, only stress levels further from the contact application should be considered. Both blade profiles feature identical levels of maximum stresses though they are not located in the same part of the blade: the initial blade profile features a maximum stress in the vicinity of its foot, along the trailing edge, while the optimized model's maximum stress is found close to its foot, in the middle along the suction side. 


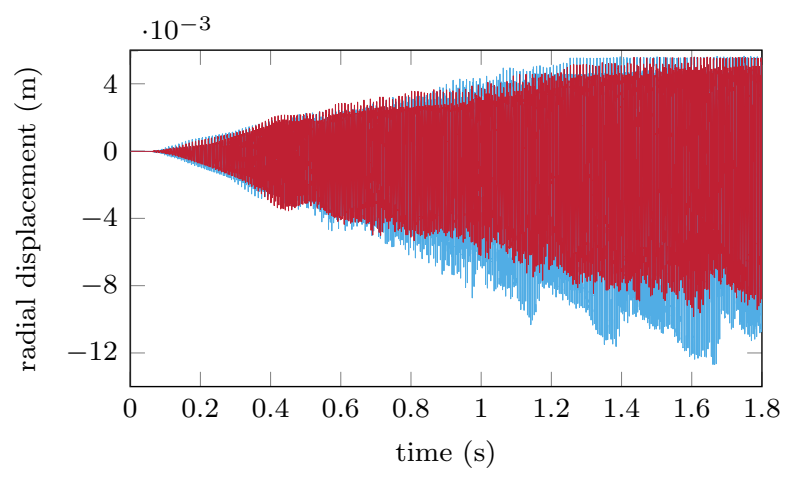

Figure 22. time history of each blade's leading edge radial displacement for interaction point $\bullet$ : initial blade (—) and optimized blade $(-)$

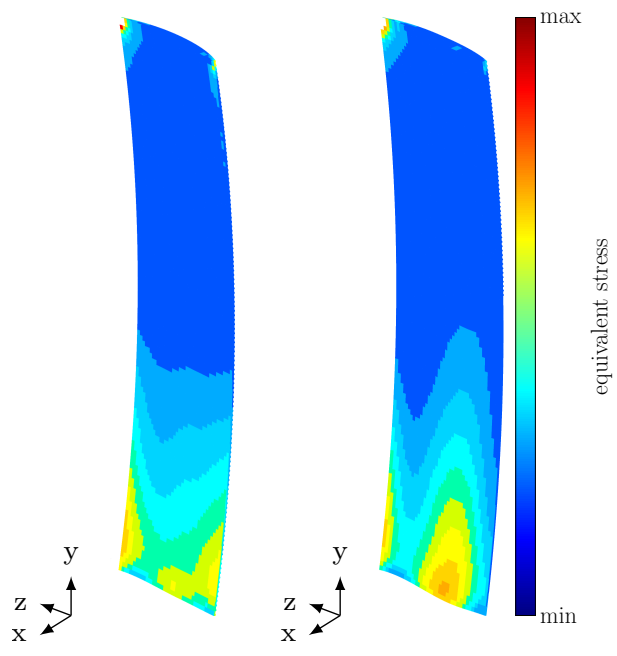

(a) initial blade

(b) optimized blade

Figure 23. normalized Von Mises stress fields during contact interaction

\section{Conclusion}

This paper presents an automated optimization procedure dedicated to bladed components subject to unilateral contacts. An original parameterization of a bladed component is proposed and validated with respect to existing blade profiles such as NACA and rotor 37. The objective-function of the optimization procedure is computed from the intrinsic clearance consumption of the blade of interest. As a first step towards a more robust strategy, aerodynamic considerations are left aside: the optimization is only based on structural aspects. The employed set of constraints can easily be extended in order to match usual design guidelines. The application of the proposed automated procedure on a NACA65-(10)10 blade profile yields an optimized blade that is found to be significantly less sensitive to contact events. Based on this first step, future work will include optimization of blades with more realistic geometries, for example with three different profiles and 3D orientations; investigation of the effect of the choice of point (leading edge or trailing edge) and mode (bending or torsion) on the results; inclusion of aerodynamic constraints in the optimization. Then, multiple objective optimization may be considered. 


\section{Acknowledgment}

This research was supported by the Natural Sciences and Engineering Research Council of Canada (NSERC).

\section{References}

[1] Ma, H., Wang, D., Tai, X., and Wen, B., 2015. "Vibration Response Analysis of Blade-Disk Dovetail Structure Under Blade Tip Rubbing Condition". Journal of Vibration and Control, 23(2), pp. 252-271. doi:10.1177/1077546315575835.

[2] Batailly, A., Agrapart, Q., Millecamps, A., and Brunel, J.-F., 2016. "Experimental and numerical simulation of a rotor/stator interaction event within an industrial high-pressure compressor". Journal of Sound and Vibration, 375, pp. 308-331. doi:10.1016/j.jsv.2016.03.016 - oai:hal-01342401.

[3] Schmiechen, P., 1997. "Travelling Wave Speed Coincidence". PhD thesis, University of London.

[4] Salvat, N., Batailly, A., and Legrand, M., 2016. "Two-dimensional modeling of unilateral contact-induced shaft precessional motions in bladed-disk/casing systems". International Journal of Non-Linear Mechanics, 78, pp. 90-104. doi:10.1016/j.ijnonlinmec.2015.10.001 - oai:hal-01223046.

[5] Seo, S.-J., Choi, S.-M., and Kim, K.-Y., 2008. "Design Optimization of a Low-Speed Fan Blade with Sweep and Lean". In Proceedings of the Institution of Mechanical Engineers, Part A: Journal of Power and Energy, Vol. 222, pp. 87-92. doi:10.1243/09576509JPE410.

[6] Sieverding, F., Ribi, B., Casey, M., and Meyer, M., 2004. "Design of Industrial Axial Compressor Blade Sections for Optimal Range and Performance". Journal of Turbomachinery, 126(2), pp. 323-331. doi:10.1115/1.1737782.

[7] Samad, A., and Kim, K. Y., 2008. "Multi-Objective Optimization of an Axial Compressor Blade". Journal of Mechanical Science and Technology, 22(5), pp. 999-1007. doi:10.1007/s12206-008-0122-5.

[8] Sommer, L., and Bestle, D., 2011. "Curvature Driven Two-Dimensional Multi-Objective Optimization of Compressor Blade Sections". Aerospace Science and Technology, 15(4), pp. 334-342. doi:10.1016/j.ast.2010.08.008.

[9] Yu, J., Ji, L., Li, W., and Yi, W., 2015. "Shape Optimization of Axial Compressor Blades Using Adjoint Method With Emphasis on Thickness Distribution". In ASME Turbo Expo 2015: Turbine Technical Conference and Exposition. GT2015-42234, doi:10.1115/GT2015-42234.

[10] Li, J., Liu, B., Yang, X., and Lu, X., 2015. "Study on Coupling Optimization Design of Aspirated Compressor Airfoil Based on Two Different Parameterization Methods". In ASME Turbo Expo 2015: Turbine Technical Conference and Exposition. GT2015-42009, doi:10.1115/GT2015-42009.

[11] Schnoes, M., and Nicke, E., 2017. "A Database of Optimal Airfoils for Axial Compressor Throughflow Design". Journal of Turbomachinery, 139(5), p. 051008. doi:10.1115/1.4035075.

[12] Goel, S., Cofer, J., and Singh, H., 1996. "Turbine Airfoil Design Optimization". In ASME 1996 International Gas Turbine and Aeroengine Congress and Exhibition, Vol. Volume 1: Turbomachinery, p. V001T01A055. 96-GT-158, doi:10.1115/96-gt-158.

[13] Chen, N., Zhang, H., Ning, F., Xu, Y., and Huang, W., 2006. "An Effective Turbine Blade Parametrization and Aerodynamic Optimization Procedure using an Improved Response Surface Method". In ASME Turbo Expo 2006: Power for Land, Sea, and Air, Vol. Volume 6: Turbomachinery, Parts A and B, pp. 1169-1180. GT2006-90104, doi:10.1115/gt2006-90104.

[14] Erler, E., Vo, H. D., and Yu, H., 2015. "Desensitization of Axial Compressor Performance and Stability to Tip Clearance Size". In ASME Turbo Expo 2015: Turbine Technical Conference and Exposition. GT2015-42746, doi:10.1115/1.4031865.

[15] Hulme, C., Fiebiger, S., and Szwedowicz, J., 2015. "Axial compressor blade failure, design modification, and its validation". In Proceedings of the ASME Turbo Expo 2015 conference, Vol. Volume 7A: Structures and Dynamics, p. V07AT28A011. GT2015-43312, doi:10.1115/gt2015-43312. 
[16] Batailly, A., Legrand, M., Millecamps, A., Cochon, S., and Garcin, F., 2014. "Redesign of a High-Pressure Compressor Blade Accounting for Nonlinear Structural Interactions". Journal of Engineering for Gas Turbines and Power, 137(2), p. 022502. doi:10.1115/1.4028263 - oai:hal-01120158.

[17] Batailly, A., and Millecamps, A., 2016. "Minimising Clearance Consumption: a Key Factor for the Design of Blades Robust To Rotor/Stator Interactions ?". In ASME Turbo Expo 2016: Turbomachinery Technical Conference and Exposition, Vol. Volume 7A: Structures and Dynamics, p. V07AT32A011. GT2016-56721, doi:10.1115/GT2016-56721 - oai:hal-01618315.

[18] Pritchard, L. J., 1985. "An Eleven Parameter Axial Turbine Airfoil Geometry Model”. In ASME 1985 International Gas Turbine Conference and Exhibit, Vol. Volume 1: Aircraft Engine; Marine; Turbomachinery; Microturbines and Small Turbomachinery, p. V001T03A058. 85-GT-219, doi:10.1115/85-gt-219.

[19] Cho, S. Y., Yoon, E. S., and Choi, B. S., 2002. "A Study on an Axial-Type 2-D Turbine Blade Shape for Reducing the Blade Profile Loss". KSME International Journal, 16(8), pp. 1154-1164. doi:10.1007/bf02984026.

[20] Ronald H., A., 2003. "Axial-Flow Compressor Blade Profiles". In Axial-Flow Compressors. ASME, ch. 4, pp. 59-76. doi:10.1115/1.801926.ch4.

[21] Reid, L., and Moore, R. D., 1978. Design and Overall Performance of Four Highly Loaded, High-Speed Inlet Stages for an Advanced High-Pressure Ratio Core Compressor. Tech. Rep. NASA TP-1337, NASA.

[22] Steinert, W., Eisenberg, B., and Starken, H., 1991. "Design and Testing of a Controlled Diffusion Airfoil Cascade for Industrial Axial Flow Compressor Application". Journal of Turbomachinery, 113(4), pp. 583-590. doi:10.1115/1.2929119.

[23] Abramson, M. A., Audet, C., Couture, G., Dennis, Jr., J. E., Le Digabel, S., and Tribes, C. The NOMAD Project. Software available online : https://www.gerad.ca/nomad/.

[24] Abramson, M. A., Audet, C., Dennis, J. E., and Digabel, S. L., 2009. "OrthoMADS: A Deterministic MADS Instance with Orthogonal Directions". SIAM Journal on Optimization, 20(2), jan, pp. 948-966. doi: $10.1137 / 080716980$.

[25] Ribes, A., and Caremoli, C., 2007. "Salomé Platform Component Model for Numerical Simulation". In 31st Annual International Computer Software and Applications Conference, Vol. 2, pp. 553-564. doi:10.1109/compsac.2007.185. 